\title{
A Problem of Coefficient Determination in Parabolic Equations Solved as Moment Problem
}

\author{
Dr. Maria B. Pintarelli \\ Grupo de Aplicaciones Matematicas y Estadisticas de la Facultad de Ingenieria (GAMEFI), UNLP, and Departamento de Matematica,
} Facultad de Ciencias Exactas, Universidad Nacional de La Plata

e-mail: mariabpintarelli@gmail.com

\begin{abstract}
The problem is to find $a(t)$ y $w(x, t)$ such that $w_{t}=a(t)\left(w_{x}\right)_{x}+r(x, t)$ under the initial condition $w(x, 0)=\varphi(x)$ and the boundary conditions $w(0, t)=0 ; w_{x}(0, t)=w_{x}(1, t)+\alpha w(1, t)$ about a region $D=\{(x, t), \quad 0<x<1, \quad t>0\}$. In addition it must be fulfilled $\int_{0}^{1} w(x, t) d x=E(t)$ where $\varphi(x), r(x, t)$ and $E(t)$ are known functions and $\alpha$ is an arbitrary real number other than zero.

The objective is to solve the problem as an application of the inverse moment problem. Will be found an approximated solution and bounds for the error of the estimated solution using the techniques on moments problem. In addition, the method is illustrated with several examples.
\end{abstract}

Keywords: generalized moment problem;integral equations;inverse problem;parabolic PDEs;truncated expansion method.

\section{Introduction}

We want to find $a(t)$ y $w(x, t)$ such that

$w_{t}=a(t)\left(w_{x}\right)_{x}+r(x, t)$

under the initial condition

$w(x, 0)=\varphi(x)$

and the boundary conditions

$w(0, t)=0 \quad w_{x}(0, t)=w_{x}(1, t)+\alpha w(1, t)$

about a region $D=\{(x, t), \quad 0<x<1, \quad t>0\}$

In addition it must be fulfilled

$\int_{0}^{1} w(x, t) d x=E(t)$

where $\varphi(x), r(x, t)$ and $E(t)$ are known functions and $\alpha$ is an arbitrary real number other than zero.

We also assume that the underlying space is $L^{2}(D)$.

This problem is studied in [1]. Citing the abstract of this work: "this paper investigates the inverse problem of simultaneously determining the time-dependent thermal diffusivity and the temperature distribution in a parabolic equation in the case of nonlocal boundary conditions containing a real parameter and integral overdetermination conditions, and under some consistency conditions on the input data the existence, uniqueness and continuously dependence upon the data of the classical solution are shown by using the generalized Fourier method".

In general the methods applied to solve the problem are varied. Other works that solve the parabolic equation but under different conditions are [2, 3, 4].

There is a great variety of inverse problems in which a parabolic equation must be solved and additionally we must determine an unknown parameter[5, 6, 7], to name a few examples.

The objective of this work is to show that we can solve the problem using the techniques of inverse moments problem. We focus the study on the numerical approximation.

First deduce an exact expression for $a(t) w(1, t)$. Then, we wrote $w^{*}(x, t)=a(t) w(x, t)$, and is resolved in a first step in numerical form the integral equation

$\iint_{D} G(x, t) x^{m} \operatorname{Exp}(-(m+1) t) d x d t=\psi 1(m)$

where $\psi 1(m)$ is written in terms of known expressions, and

$G(x, t)=-x w_{x}^{*}(x, t)-w_{t}^{*}(x, t)$

it is the function to be determined.

In a second step the following integral equation is solved in 
numerical form

$\iint_{D} w^{*}(x, t) K(m, n, x, t) d x d t=\psi 2(m, n)$

where $w^{*}(x, t)$ it's the unknown function, $\psi 2(m, n)$ is an expression in function of $G(x, t)$ with $K(m, n, x, t)$ known.

Both integral equations are solved numerically by applying the moment problems techniques.

Then we find an approximation for $w(x, t)$, this approximation we write $w A p(x, t)$, using the solution found in the second step and condition (3).

Finally we find an approximation for $a(t)$ using $a(t) w(1, t)$ and $w A p(x, t)$.

\section{Inverse Generalized Moment Problem}

The d-dimensional generalized moment problem $[8,9,10]$ and $[11,12]$ can be posed as follows: find a function $f$ on a domain $\Omega \subset \mathbf{R}^{d}$ satisfying the sequence of equations

$\int_{\Omega} f(x) g_{i}(x) d x=\mu_{i} \quad i \in \mathbf{N}$

where $\left(g_{i}\right)$ is a given sequence of functions lying in $\mathbf{L}^{2}(\Omega)$ linearly independent, and the sequence of real numbers $\left\{\mu_{i}\right\}_{i \in N}$ are the known data.

The moments problem of Hausdorff is a classic example of moments problem, is to find a function $f(x)$ in $(a, b)$ such that

$\mu_{i}=\int_{a}^{b} x^{i} f(x) d x \quad i \varepsilon N$

In this case $g_{i}(x)=x^{i} \quad i \varepsilon N$. If the interval of integration is $(0, \infty)$ we have the problem of moments of Stieltjes, if the interval of integration is $(-\infty, \infty)$ we have the problem of moments of Hamburger.

It can be proved that [12] a necessary and sufficient condition for the existence of a solution of (4) is that $\sum_{i=1}^{\infty}\left(\sum_{j=1}^{i} C_{i j} \mu_{j}\right)^{2}<\infty$ where $C_{i j}$ are given by (11) and (12).

Moment problem are usually ill-posed in the sense that there may be no solution and if there is no continuous dependence on the given data. There are various methods of constructing regularized solutions, that is, approximate solutions stable with respect to the given data. One of them is the method of truncated expansion.

The method of truncated expansion consists in approximating (4) by finite moment problems

$\int_{\Omega} f(x) g_{i}(x) d x=\mu_{i} \quad i=1,2, \ldots, n$

and consider as an approximate solution of $f(x)$ to $p_{n}(x)=\sum_{i=0}^{n} \lambda_{i} \varphi_{i}(x)$. The $\varphi_{i}(x)$ result from ortonormalize $\left\langle g_{1}, g_{2}, \ldots, g_{n}\right\rangle$ and $\lambda_{i}$ are coefficients as a function of the $\mu_{i}$. Solved in the subspace $\left\langle g_{1}, g_{2}, \ldots, g_{n}\right\rangle$ generated by $g_{1}, g_{2}, \ldots, g_{n}$ (5) is stable. Considering the case where the data $\mu=\left(\mu_{1}, \mu_{2}, \ldots, \mu_{n}\right)$ are inexact, convergence theorems and error estimates for the regularized solutions they are applied.

\section{Resolution of the Parabolic Partial Differen- tial Equation}

We consider the equation $w_{t}=a(t)\left(w_{x}\right)_{x}+r(x, t)$. If we integrate with respect to $x$ between 0 and 1 we obtain

$\int_{0}^{1} w_{t} d x=a(t)\left[w_{x}(1, t)-w_{x}(0, t)\right]+\int_{0}^{1} r(x, t) d x$

If we write $r^{*}(t)=\int_{0}^{1} r(x, t) d x$ and $E^{\prime}(t)=\frac{d}{d t} E(t)$ then

$E^{\prime}(t)=a(t)(-\alpha w(1, t))+r^{*}(t)$

Thus

$a(t) w(1, t)=\frac{r^{*}(t)-E^{\prime}(t)}{\alpha}$

On the other hand we consider the vector field

$F^{*}=\left(a(t) w_{x},-a(t) w\right)=\left(w_{x}^{*},-w^{*}\right)$

Let $u(i, z, x, t)$ be the auxiliary function

$u(i, z, x, t)=x^{i} e^{-(z+1) t}$

Then

$$
\begin{array}{r}
\operatorname{div}\left(u F^{*}\right)=\left(u a(t) w_{x}\right)_{x}-(u a(t) w)_{t}= \\
=u_{x} a(t) w_{x}+u a(t) w_{x x}-u_{t} a(t) w-u a^{\prime}(t) w-u a(t) w_{t}
\end{array}
$$

Also

$u d i v\left(F^{*}\right)=u a(t) w_{x x}-u a^{\prime}(t) w-u a(t) w_{t}$

Moreover, as

$u \operatorname{div}\left(F^{*}\right)=\operatorname{div}\left(u F^{*}\right)-F^{*} \cdot \nabla u$

$\iint_{D} u \operatorname{div}\left(F^{*}\right) d A=\iint_{D} \operatorname{div}\left(u F^{*}\right) d A-\iint_{D} F^{*} \nabla u d A$

where $\nabla u=\left(u_{x}, u_{t}\right)$

besides

$$
\begin{aligned}
& \iint_{D} \operatorname{div}\left(u F^{*}\right) d A=\iint_{D}\left(\left(u w_{x}^{*}\right)_{x}-\left(u w^{*}\right)_{t}\right) d A= \\
& =\iint_{D} u \operatorname{div}\left(F^{*}\right) d A+\iint_{D}\left(\left(u_{x} w_{x}^{*}\right)-\left(u_{t} w^{*}\right)\right) d A
\end{aligned}
$$

Then of (7) and (8)

$\iint_{E}\left(u_{x} w_{x}^{*}-u_{t} w^{*}\right) d A=\iint_{E} F^{*} \nabla u d A$

Can be proven that, after several calculations, (9) is written as 


$$
\begin{array}{r}
\int_{0}^{1} w^{*}(x, 0) u(i, z, x, 0) d x- \\
\frac{z+1}{i+1} \int_{0}^{\infty}\left(w^{*}(1, t) u(i, z, 1, t)-w^{*}(0, t) u(i, z, 0, t)\right) d t= \\
=-\frac{z+1}{i+1} \int_{0}^{1} \int_{0}^{\infty} w_{x}^{*}(x, t) u d t d x- \\
\int_{0}^{1} \int_{0}^{\infty} w_{t}^{*}(x, t) u d t d x
\end{array}
$$

and if $z=i$ then

$$
\begin{array}{r}
\int_{0}^{1} \int_{0}^{\infty}\left(-x w_{x}^{*}(x, t)-w_{t}^{*}(x, t)\right) u(i, i, x, t) d t d x= \\
\int_{0}^{1} w^{*}(x, 0) u(i, i, x, 0) d x- \\
-\int_{0}^{\infty}\left(w^{*}(1, t) u(i, i, 1, t)=\varphi 1(i)\right.
\end{array}
$$

Note that

$w^{*}(x, 0)=a(0) w(x, 0)=a(0) \varphi(x)$

$a(0)=\frac{r^{*}(0)-E^{\prime}(0)}{\alpha \varphi(1)}$

and

$w^{*}(1, t)=a(t) w(1, t)$

previously calculated

We wrote $G(x, t)=-x w_{x}^{*}(x, t)-w_{t}^{*}(x, t)$

We solve the integral equation numerically

$\int_{0}^{1} \int_{0}^{\infty} G(x, t) u(i, i, x, t) d t d x=$

$=\int_{0}^{1} \int_{0}^{\infty} G(x, t) H_{i}(x, t) d t d x=\varphi 1(i)=\mu_{i}$

and we will obtain an approximate solution for $G(x, t)$

We can apply the truncated expansion method detailed in [11] and generalized in $[12,13]$ to find an approximation $p_{1 n}(x, t)$ for $G(x, t)$ for the corresponding finite problem with $i=0,1, \ldots n$ where $n$ is the number of moments $\mu_{i}$.

We consider the base $\phi_{i}(x, t) \quad i=0,1,2, \ldots$ obtained by applying the Gram-Schmidt orthonormalization process on $H_{i}(x, t) \quad i=0,1,2, \ldots n$ and adding to the resulting set the necessary functions until reaching an orthonormal basis.

We approach the solution $G(x, t)$ with $[12,13]$ :

$$
\begin{array}{r}
p_{1 n}(x, t)=\sum_{i=0}^{n} \lambda_{i} \phi_{i}(x, t) \\
\lambda_{i}=\sum_{j=0}^{i} C_{i j} \mu_{j} \quad i=0,1,2, \ldots, n
\end{array}
$$

And the coefficients $C_{i j}$ verifies

$C_{i j}=\left(\sum_{k=j}^{i-1}(-1) \frac{\left\langle H_{i}(x, t) \mid \phi_{k}(x, t)\right\rangle}{\left\|\phi_{k}(x, t)\right\|^{2}} C_{k j}\right) \cdot\left\|\phi_{i}(x, t)\right\|^{-1}$

$$
1<i \leq n ; 1 \leq j<i
$$

The terms of the diagonal are

$$
C_{i i}=\left\|\phi_{i}(x, t)\right\|^{-1} \quad i=0,1, \ldots, n .
$$

The proof of the following theorem is in $[14,15]$. In [15] he proof is done for $b_{2}$ finite. If $b_{2}=\infty$ instead of taking polynomials the Legendre are taken polynomials of Laguerre. In [16] the demonstration is done for the one-dimensional case.

Theorem. Let $\left\{\mu_{i}\right\}_{i=0}^{n}$ be a set of real numbers and suppose that $f(x, t) \in L^{2}\left(\left(a_{1}, b_{1}\right) \times\left(a_{2}, \infty\right)\right)$ verify for some $\varepsilon$ and $E$ (two positive numbers)

$$
\begin{aligned}
\sum_{i=0}^{n}\left|\int_{a_{2}}^{\infty} \int_{a_{1}}^{b_{1}} H_{i}(x, t) f(x, t) d x d t-\mu_{i}\right|^{2} & \leq \varepsilon^{2} \\
\int_{a_{2}}^{\infty} \int_{a_{1}}^{b_{1}}\left(x f_{x}^{2}+t f_{t}^{2}\right) e^{x+t} d x d t & \leq E^{2}
\end{aligned}
$$

then

$$
\begin{array}{r}
\int_{a_{2}}^{\infty} \int_{a_{1}}^{b_{1}}|f(x, t)|^{2} d x d t \leq \\
\min _{i}\left\{\left\|C C^{T}\right\| \varepsilon^{2}+\frac{E^{2}}{8(i+1)^{2}} ; i=0,1, \ldots, n\right\}
\end{array}
$$

where $C$ is the triangular matrix with elements $C_{i j} \quad(1<i \leq$ $n ; 1 \leq j<i)$.

and

$\int_{a_{2}}^{\infty} \int_{a_{1}}^{b_{1}}\left|p_{1 n}(x, t)-f(x, t)\right|^{2} d x d t \leq\left\|C C^{T}\right\| \varepsilon^{2}+\frac{E^{2}}{8(n+1)^{2}}$

It must be fulfilled that

$t^{i} f(x, t) \longrightarrow 0 \quad$ if $t \longrightarrow \infty \quad \forall i \varepsilon N$

If we apply the truncated expansion method to solve equation (10) we obtain an approximation $p_{1 n}(x, t)$ for $G(x, t)=-x w_{x}^{*}(x, t)-w_{t}^{*}(x, t)$.

Then we have an equation in first order partial derivatives

$-x w_{x}^{*}(x, t)-w_{t}^{*}(x, t)=p_{1 n}(x, t)$

of the form

$A_{1}(x, t) w_{x}^{*}(x, t)+A_{2}(x, t) w_{t}^{*}(x, t)=p_{1 n}(x, t)$ 
where $A_{1}(x, t)=-x$ and $A_{2}(x, t)=-1$. It is solved as in [15], ie, we can prove that solving this equation is equivalent to solving the integral equation

$\int_{0}^{1} \int_{0}^{\infty} w^{*}(x, t) K(i, z, x, t) d t d x=\varphi 2(i, z)=\mu_{i z}$

where

$$
\begin{array}{r}
K(i, z, x, t)=\left[\left(x\left(A_{1}\right)_{x}(x, t)+\right.\right. \\
\left.\left(A_{2}\right)_{t}(x, t)+i A_{1}(x, t)-x(z+1) A_{2}(x, t)\right] x^{i-1} e^{-(z+1) t}= \\
=(z-i) x^{i} e^{-(z+1) t}
\end{array}
$$

that is

$\int_{0}^{1} \int_{0}^{\infty} u(i, z, x, t) w^{*}(x, t)(z-i) d t d x=\varphi 2(i, z)=\mu_{i z}$

with

$$
\begin{array}{r}
\varphi 2(i, z)=\int_{0}^{\infty}\left[u(i, z, 1, t) w^{*}(1, t)\right] d t- \\
-\int_{0}^{1} u(i, z, x, 0) w^{*}(x, 0) d x-\int_{0}^{1} \int_{0}^{\infty} u p_{1 n} d t d x
\end{array}
$$

Again we consider the base $\phi_{i z}(x, t) \quad i=0,1,2, . . ; z=$ $i+1, \ldots \ldots$ obtained by applying the Gram-Schmidt orthonormalization process on $u(i, z, x, t)(z-i)=K_{i z}(x, t) \quad i=$ $0,1,2, \ldots ; z=i+1, \ldots$. and then the above equation can be transformed into a generalized moment problem

$\int_{0}^{1} \int_{0}^{\infty} w^{*}(x, t) K_{i z}(x, t) d t d x=\mu_{i z}$

Applying again the techniques of generalized moments problem to the corresponding finite problem, we found an approximate solution $p_{2 n}(x, t)$ for $w^{*}(x, t)$.

To find a numerical approximation for $w(x, t)$ we use condition (3):

$$
\begin{aligned}
& \int_{0}^{1} a(t) w(x, t) d x \approx \int_{0}^{1} p_{2 n}(x, t) d x=p_{3}(t) \\
& \therefore \quad a(t) E(t) \approx p_{3}(t)
\end{aligned}
$$

Then

$$
w(x, t) \approx \frac{p_{2 n}(x, t)}{\frac{p_{3}(t)}{E(t)}}=w A p(x, t)
$$

And a numerical approximation for $a(t)$ will be

$$
a(t) \approx \frac{a(t) w(1, t)}{w A p(1, t)}=a A p(t)
$$

We can measure the accuracy of the approximation (13) using the previous theorem, where $\mu_{i}$ would be the ith generalized moment of $w A p(x, t)$, that is, we consider the moments of $w(x, t)$ measured with error.

An analogous argument is used to measure the accuracy of the approximation $a A p(t)$

\section{Numerical Examples}

To obtain an approximation $p_{1 n}(x, t)$ for $G(x, t)=$ $-x w_{x}^{*}(x, t)-w_{t}^{*}(x, t)$, we consider the base $\phi_{i}(x, t) \quad i=$ $0,1,2, \ldots n$ obtained by applying the Gram-Schmidt orthonormalization process on $H_{i}(x, t)=x^{i} e^{-(i+1) t} \quad i=0,1,2, \ldots n$.

In other words, it applies the Gram-Schmidt orthonormalization process on $\left\{e^{-t}, x e^{-2 t}, x^{2} e^{-3 t}, \ldots, x^{n-1} e^{-n t}\right\}$, and is taken as a measure $\iint_{D} \cdot e^{-t} d t d x$.

We will obtain, by applying the truncated expansion method, $p_{1 n}^{*}(x, t)$ so that $e^{t} p_{1 n}^{*}(x, t)=p_{1 n}(x, t)$.

Analogously to obtain $p_{2 n}(x, t)$, we consider the base $\phi_{i z}(x, t) \quad i=0,1,2, \ldots, n_{1}, ; z=i+1, \ldots, n_{2}$ obtained by applying the Gram-Schmidt orthonormalization process on $u(i, z, x, t)(z-i)=K_{i z}(x, t) \quad i=0,1,2, \ldots, n_{1} ; z=i+1, \ldots, n_{2}$, and is taken as a measure $\iint_{D} \cdot e^{-2 t} d t d x$.

We will obtain, by applying the truncated expansion method, $p_{2 n}^{*}(x, t)$ so that $e^{2 t} p_{2 n}^{*}(x, t)=p_{2 n}(x, t)$.

To apply the method must be $w(1,0) \neq 0$.

It may happen that (13) or (14) have discontinuities because the denominator is overridden for certain values of $t$. In this case we can vary the number of moments that are taken so that the denominator does not have real roots that cancel it.

\subsection{Example 1}

We consider the equation

$$
\begin{gathered}
w_{t}=a(t)\left(w_{x}\right)_{x}+\frac{e^{\frac{-t-4 x}{8}}\left(-4-t^{3}+e^{\frac{x}{2}}\left(2+t^{3}\right)\right)}{8\left(2+t^{3}\right)} \\
0<x<1 \quad ; \quad t>0
\end{gathered}
$$

and conditions

$E(t)=(-2+\sqrt{e}) e^{-\left(\frac{1}{2}+\frac{t}{8}\right)} \quad ; \quad \alpha=\frac{1}{2} \quad ; \quad w(x, 0)=e^{-\frac{x}{2}}-1$

The following conditions are met:

$$
w(0, t)=0 \quad ; \quad w_{x}(0, t)-w_{x}(1, t)=\frac{1}{2} w(1, t)
$$

the solution is

$w(x, t)=\left(e^{-\frac{x}{2}}-1\right) e^{-\frac{t}{8}} \quad$ and $\quad a(t)=\frac{1}{2+t^{3}}$

We calculate $p_{1 n}(x, t)$ with $n=10$ moments and $p_{2 n}(x, t)$ with $n=n_{1} \times n_{2}=4 \times 4=16$ moments.

Approximates $w(x, t)$ with $w A p(x, t)$

Accuracy is $\int_{0}^{1} \int_{0}^{\infty}|w(x, t)-w A p(x, t)|^{2} d t d x=0.127967$.

And approximates $a(t)$ with $a A p(t)$

Accuracy is $\int_{0}^{\infty}|a(t)-a A p(t)|^{2} d t d x=0.0684834$. In Fig. 1 and Fig. 2 the exact solution and the approximate solution are compared. 


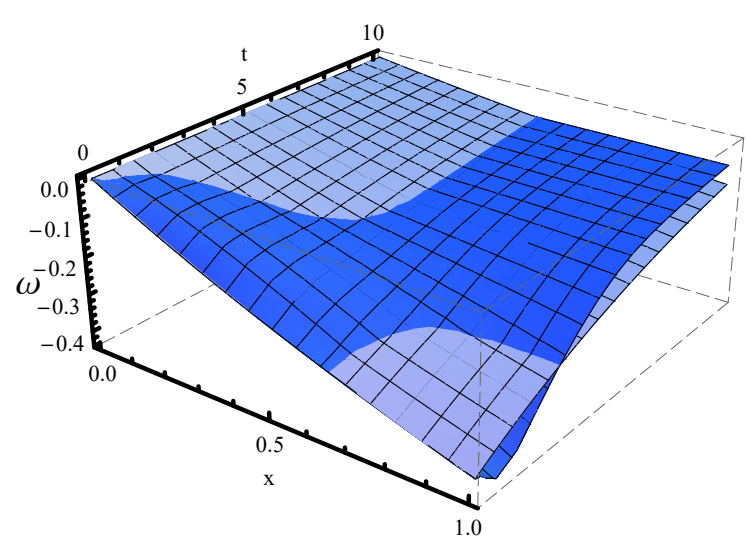

Figure 1: $w(x, t)$ and $w A p(x, t)$

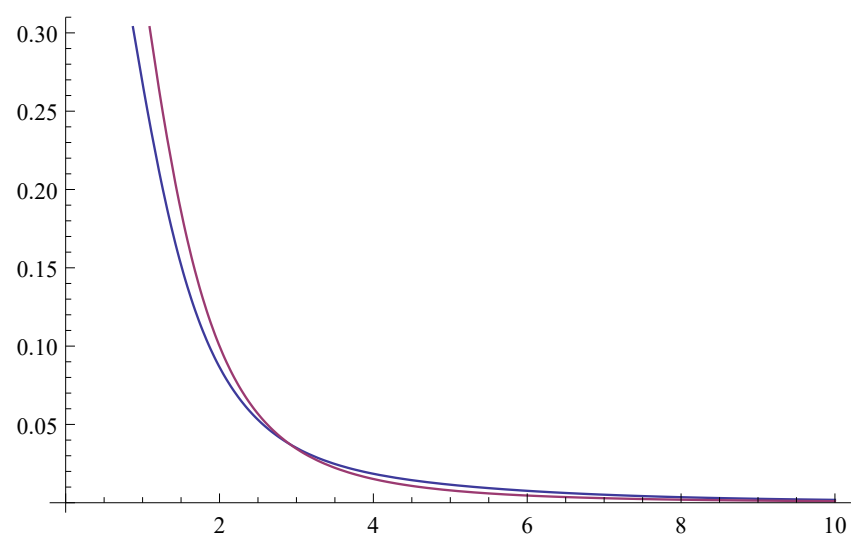

Figure 2: $a(t)$ and $a A p(t)$

\subsection{Example 2}

We consider the equation

$$
\begin{gathered}
w_{t}=a(t)\left(w_{x}\right)_{x}+\frac{-2 e^{-t}\left(2+t^{2}+e^{t} t x^{2}\right)}{\left(2+t^{2}\right)^{2}} \\
0<x<1 \quad ; \quad t>0
\end{gathered}
$$

and conditions

$E(t)=\frac{1}{6+3 t^{2}} \quad ; \quad \alpha=-2 \quad ; \quad w(x, 0)=\frac{x^{2}}{2}$

The following conditions are met:

$w(0, t)=0 \quad ; \quad w_{x}(0, t)-w_{x}(1, t)=-2 w(1, t)$

the solution is

$w(x, t)=\frac{x^{2}}{2+t^{2}} \quad$ and $\quad a(t)=e^{-t}$

We calculate $p_{1 n}(x, t)$ with $n=7$ moments and $p_{2 n}(x, t)$ with $n=n_{1} \times n_{2}=3 \times 3=9$ moments.

Approximates $w(x, t)$ with $w A p(x, t)$. Accuracy is $\int_{0}^{1} \int_{0}^{\infty}|w(x, t)-w A p(x, t)|^{2} d t d x=0.0176301$.

And approximates $a(t)$ with $a A p(t)$. Accuracy is $\int_{0}^{\infty} \mid a(t)-$ $\left.a A p(t)\right|^{2} d t d x=0.0195688$. In Fig. 3 and Fig.4 the exact solution and the approximate solution are compared.

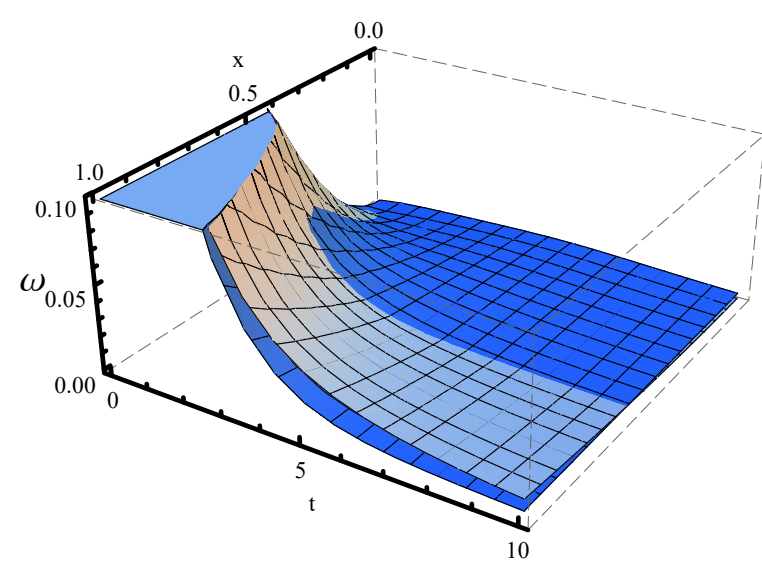

Figure 3: $w(x, t)$ and $w A p(x, t)$

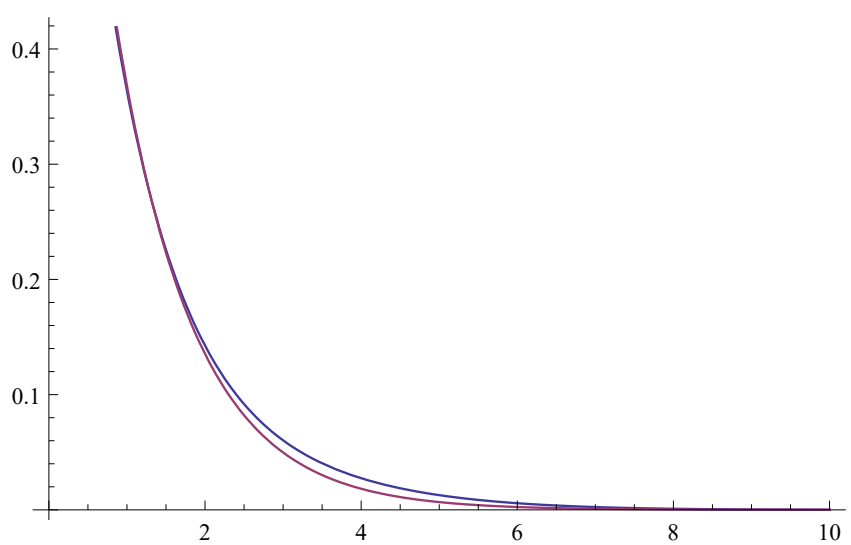

Figure 4: $a(t)$ and $a A p(t)$

\subsection{Example 3}

We consider the equation

$$
\begin{gathered}
w_{t}=a(t)\left(w_{x}\right)_{x}+\left(-\frac{1}{4} e^{-3 t}\left(4 e^{2 t}-\pi^{2}\right) \sin \left(\frac{\pi x}{2}\right)\right) \\
0<x<1 \quad ; \quad t>0
\end{gathered}
$$

and conditions

$E(t)=\frac{2 e^{-t}}{\pi} \quad ; \quad \alpha=\frac{\pi}{2} \quad ; \quad w(x, 0)=\sin \left(\frac{x \pi}{2}\right)$

The following conditions are met:

$w(0, t)=0 \quad ; \quad w_{x}(0, t)-w_{x}(1, t)=\frac{\pi}{2} w(1, t)$

the solution is

$w(x, t)=\sin \left(\frac{x \pi}{2}\right) e^{-t} \quad$ and $\quad a(t)=e^{-2 t}$

We calculate $p_{1 n}(x, t)$ with $n=7$ moments and $p_{2 n}(x, t)$ with $n=n_{1} \times n_{2}=3 \times 3=9$ moments.

Approximates $w(x, t)$ with $w A p(x, t)$. Accuracy is $\int_{0}^{1} \int_{0}^{\infty}|w(x, t)-w A p(x, t)|^{2} d t d x=0.0916382$.

And approximates $a(t)$ with $a A p(t)$.

Accuracy is $\int_{0}^{\infty}|a(t)-a A p(t)|^{2} d t d x=0.188199$. In Fig.5 and Fig. 6 the exact solution and the approximate solution are compared. 


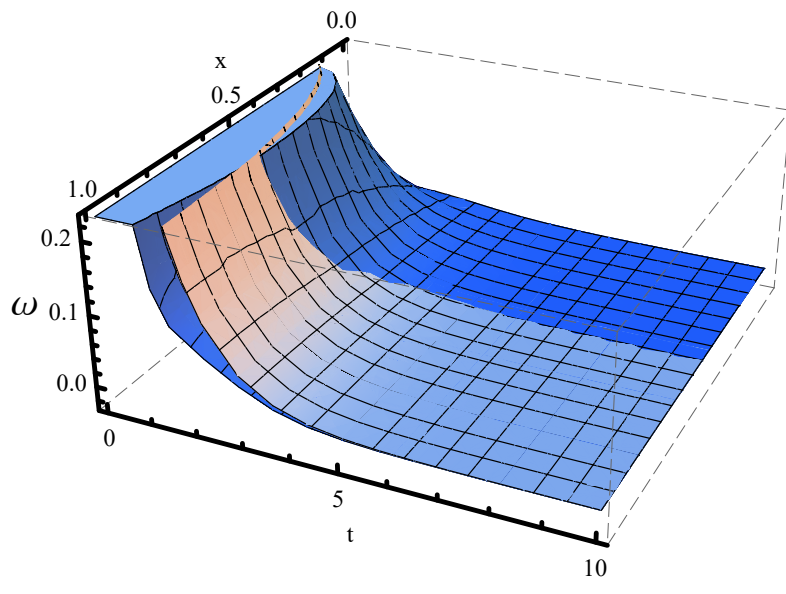

Figure 5: $w(x, t)$ and $w A p(x, t)$

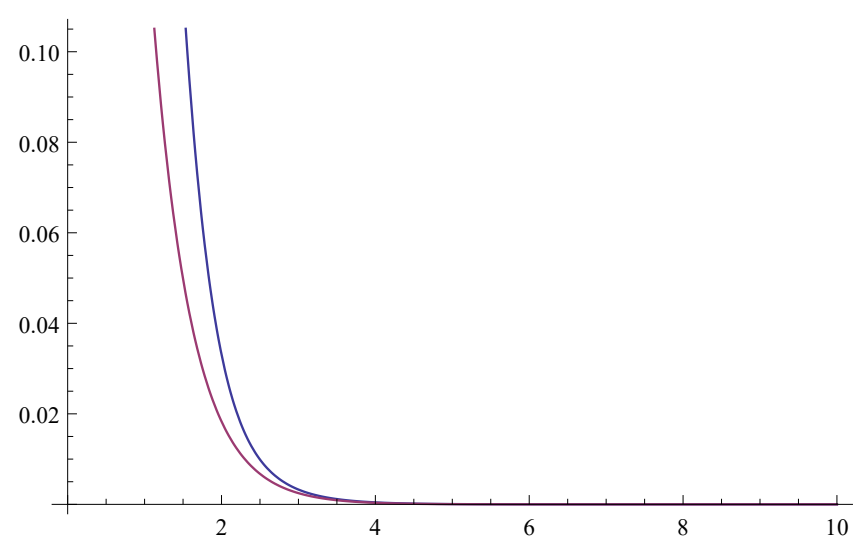

Figure 6: $a(t)$ and $a A p(t)$

\section{Conclusion}

We consider the problem of finding $a(t)$ y $w(x, t)$ such that

$w_{t}=a(t)\left(w_{x}\right)_{x}+r(x, t)$

under the initial condition $w(x, 0)=\varphi(x)$ and the boundary conditions $w(0, t)=0$ and $w_{x}(0, t)=w_{x}(1, t)+\alpha w(1, t)$ about a region $D=\{(x, t), \quad 0<x<1, \quad t>0\}$. In addition it must be fulfilled $\int_{0}^{1} w(x, t) d x=E(t)$ where $\varphi(x), r(x, t)$ and $E(t)$ are known functions and $\alpha$ is an arbitrary real number other than zero. We also assume that the underlying space is $L^{2}(D)$. First deduce an exact expression for $a(t) w(1, t)$. Then, we wrote $w^{*}(x, t)=a(t) w(x, t)$, and is resolved in a first step in numerical form the integral equation

$\iint_{D} G(x, t) x^{m} \operatorname{Exp}(-(m+1) t) d x d t=\psi 1(m)$

where

$G(x, t)=-x w_{x}^{*}(x, t)-w_{t}^{*}(x, t)$

it is the function to be determined.

In a second step the following integral equation is solved in numerical form

$\iint_{D} w^{*}(x, t) K(m, n, x, t) d x d t=\psi 2(m, n)$

where $w^{*}(x, t)$ it's the unknown function, $\psi 2(m, n)$ is an expression in function of $G(x, t)$ with $K(m, n, x, t)$ known.
Both integral equations are solved numerically by applying the moment problems techniques.

Then we find an approximation for $w(x, t)$, this approximation we write $w A p(x, t)$, using the solution found in the second step and condition $\int_{0}^{1} w(x, t) d x=E(t)$.

Finally we find an approximation for $a(t)$ using $a(t) w(1, t)$ and $w A p(x, t)$.

\section{Acknowledgement}

My thanks to the Faculty of Engineering and to the Faculty of Exact Sciences of the National University of La Plata, Argentina.

\section{References}

[1] Oussaeif Taki-Eddine and Bouzziani Abdelfatah, "An Inverse Coefficient Problem for a Parabolic Equation under Nonlocal Boundary and Integral Overdetermination Conditions",International Journal of Partial Differential Equations and Applications, Vol. 2, No 3, (2014), pp.38-43.

[2] W. Liao, M. Dehghan, and A. Mohebbi, "Direct numerical method for an inverse problem of a parabolic partial differential equation", $J$. Comput. Appl. Math, 232, (2009), pp.351-360.

[3] J.R. Cannon, Y. Lin, and S. Wang, " Determination of a control parameter in a parabolic partial differential equation", J. Austral. Math. Soc. Ser. B., 33 ,(1991), pp.149-163.

[4] Nadiya Huzyk,'Inverse problem of determinig the coefficients in a degenerate parabolic equation", Electronic Journal of Dierential Equations, Vol. 2014 , No. 172, (2014), pp.1-11.

[5] Baiyu Wang, Anping Liao and Wei Liu, "Simultaneous determination of unknown two parameters in parabolic equation", International Journalof Applied Mathematics and Computation, Vol. 4, (2012), pp.332336.

[6] J. Biazar, T. Houlari, "Implementation of Sinc-Galerkin on Parabolic Inverse problem with unknown boundary condition"Int. J. Industrial Mathematics, Vol. 7, No. 4, (2015), pp.313-319.

[7] Mehdi Dehghan and Mehdi Tatari, "Determination of a control parameter in a one-dimensional parabolic equation using the method of radial basis functions",Mathematical and Computer Modelling, 44, (2006), pp.1160-1168.

[8] Akheizer N I, The classical moment problem, Olivier and Boyd, Edinburgh, 1965

[9] Akheizer N I and Krein M G, Some questions in the theory of moment,Am. Math. Soc. Providence, RI, 1962.

[10] Shohat J A and Tamarkin J D, The problem of Moments, Math. Surveys,Am. Math. Soc., Providence, RI, 1943.

[11] G. Talenti, "Recovering a function from a finite number of moments", Inverse Problems, 3 ,(1987), pp.501-517.

[12] D.D. Ang, R. Goreno, V.K. Le and D.D. Trong,Moment theory and some inverse problems in potential theory and heat conduction, Lectures Notes in Mathematics, Springer-Verlag, Berlin, 2002.

[13] M.B. Pintarelli and F. Vericat, "Stability theorem and inversion algorithm for a generalized moment problem", Far East Journal of Mathematical Sciences, 30,(2008), pp.253-274.

[14] M.B. Pintarelli and F. Vericat, "Bi-dimensional inverse moment problems",Far East Journal of Mathematical Sciences, 54, (2011), pp.1-23.

[15] M.B.Pintarelli, "Linear partial differential equations of first order as bi-dimensional inverse moment problem" Applied Mathematics, Vol. 6, No 6, (2015), pp.979-989.

[16] M.B.Pintarelli, "Parabolic partial differential equations as inverse moments problem",Applied Mathematics, Vol. 7, No 1, (2016),pp.7799 